\title{
NOTA
}

\section{CARACTERÍSTICAS QUÍMICAS DO LIXO DE FORMIGUEIROS DE Atta sexdens rubropilosa (HYMENOPTERA: FORMICIDAE) MANTIDOS COM DIFERENTES SUBSTRATOS ${ }^{(1)}$}

\author{
Marcelo Braga Bueno Guerra ${ }^{(2)}$, Carlos Ernesto G.R. Schaefer $^{(3)} \&$ \\ Leandro Sousa-Souto ${ }^{(4)}$
}

\begin{abstract}
RESUMO
As saúvas (Atta spp.) estão amplamente distribuídas pelo continente SulAmericano e são consideradas importantes componentes do ecossistema neotropical. Diversos estudos têm demonstrado o efeito de formigueiros no enriquecimento do solo e na facilitação ao estabelecimento de espécies vegetais. Possivelmente o enriquecimento do solo seja devido ao acúmulo de matéria orgânica decomposta em câmaras de descarte (lixo) no interior das colônias. Entretanto, pouco se sabe sobre a composição química do lixo de formigas cortadeiras. $O$ objetivo do presente trabalho foi comparar os teores de nutrientes do lixo produzido por colônias de Atta sexdens rubropilosa Forel (Hymenoptera: Formicidae), em laboratório, mantidas com folhas de duas espécies de plantas. Os tratamentos foram dois tipos de substrato (Acalypha sp. ou Bauhinia sp.), repetidos quatro vezes. Oito colônias foram divididas em dois grupos $(n=4)$, e cada grupo foi mantido com apenas um tratamento. Após 30 dias de experimento, as amostras de folhas e lixo foram secas em estufa $\left(70^{\circ} \mathrm{C}\right)$ e submetidas à digestão ácida, para determinação das concentrações de N, P, K, S, Ca e Mg. Diferenças nas concentrações dos macronutrientes entre os tratamentos (lixo ou folha) foram comparadas por meio de ANOVA e teste T. As concentrações de nutrientes no lixo foram sempre maiores do que nas folhas, independentemente da espécie cortada. Folhas de Acalypha sp. apresentaram concentrações significativamente maiores do que folhas de Bauhinia sp. com relação aos teores de $\mathrm{P}, \mathrm{Ca}, \mathrm{Mg}$ e S; entretanto, as concentrações do lixo em todos os formigueiros foram muito próximas, indicando incorporação adicional de nutrientes por meio do fungo, excreções e, ou, cadáveres no lixo. Esses resultados indicam que, independentemente do substrato cortado, o lixo produzido apresenta maiores concentrações de nutrientes do que as folhas, sendo importante locus de
\end{abstract}

\footnotetext{
(1) Parte do Projeto de Pesquisa do primeiro autor, financiado pela FAPEMIG. Recebido para publicação em outubro de 2006 e aprovado em maio de 2007.

${ }^{(2)}$ Graduando em Química pela Universidade Federal de Viçosa - UFV. CEP 36570-000 Viçosa (MG). E-mail: marcelobbg@gmail.com

(3) Professor do Departamento de Solos, UFV. E-mail: carlos.schaefer@ufv.br

(4) Doutorando em Entomologia, UFV. E-mail: leandrosouto@insecta.ufv.br
} 
reciclagem de nutrientes no ecossistema. $O$ lixo pode ser uma das principais razões para o aumento da concentração de nutrientes em solos de formigueiros.

Termos de indexação: ciclagem de nutrientes, saúvas, fertilidade do solo, matéria orgânica.

\title{
SUMMARY: CHEMICAL CHARACTERISTICS OF NEST REFUSE OF Atta sexdens rubropilosa (HYMENOPTERA: FORMICIDAE) REARED WITH DIFFERENT SUBSTRATES
}

\begin{abstract}
Leaf-cutting ants (Atta spp.) are widely distributed in South America, and are considered to be important components of the neotropical ecosystems. Several studies have demonstrated the effect of ant-nesting in soil enrichment, thus facilitating vegetation succession. Possibly, this enrichment is due to decomposed organic matter concentration after disposal of nest refuse in the deep soil by the colonies. However, little is known about the chemical composition of refuse material produced by leaf-cutting ants. The present work aimed to compare the macronutrient concentration in nest refuse and leaves of two different harvested plants (Acalypha sp. or Bauhinia sp.) in colonies of Atta sexdens rubropilosa Forel (Hymenoptera: Formicidae) maintained in laboratory. Eight colonies were divided into two groups $(n=4)$, and each one was maintained with only one feed type. After 30 days of experiment, samples of leaves and refuse were oven-dried at $70{ }^{\circ} \mathrm{C}$ and subjected to acid digestion for chemical determination of total $\mathrm{N}, \mathrm{P}, \mathrm{K}, \mathrm{S}, \mathrm{Ca}$ and $\mathrm{Mg}$. Differences in concentrations of macronutrients in both leaves and refuse were compared using ANOVA and T test. Nutrient concentration in the refuse material was consistently higher than in leaves, for both plants. Acalypha sp. leaves showed greater nutrient concentration than those of Bauhinia sp., while the concentration of all refuses were very similar. This indicates an additional enrichment of nutrients in the refuse material by either ant carcasses, fungus cycling or excretions. Results suggest that nests of leaf-cutting ant are important loci of nutrient recycling of the ecosystem. Moreover, the refuse may represent an important factor for chemical enrichment in soils influenced by leaf-cutting ants.
\end{abstract}

Index terms: nutrient cycling, leaf-cutting ants, soil fertility, organic matter.

\section{INTRODUÇÃO}

As formigas cortadeiras dos gêneros Atta e Acromyrmex estão amplamente distribuídas pelo continente Sul-Americano e são consideradas importantes componentes do ecossistema neotropical (Hölldobler \& Wilson, 1990). Pelo fato de cortarem material vegetal e o transportarem para o interior de suas colônias, as saúvas tornam-se importantes agentes de acumulação de matéria orgânica no solo por meio de suas câmaras de descarte (lixo). Esse material acumulado é oriundo do processamento do fungo simbionte e pode se tornar uma fonte de nutrientes, sendo possível sua reabsorção pelas raízes das plantas por meio da ciclagem biogeoquímica de elementos. Entretanto, pouco se conhece a respeito das propriedades químicas do lixo, bem como sobre sua incorporação e mineralização nos solos. Trabalhos recentes têm enfocado a importância do fungo simbionte como fonte de $\mathrm{C}$ e N (Abril \& Bucher, 2004) e o balanço nutricional entre o fungo e o material de descarte para as operárias da colônia (Bucher et al.,
2004); contudo, poucos estudos associam o conteúdo das câmaras de lixo à dinâmica de nutrientes nos solos (Moutinho et al., 2003; Verchot et al., 2003).

Este trabalho teve por objetivo comparar as concentrações foliares de macronutrientes em duas espécies de plantas (Acalypha e Bauhinia sp.) e no lixo produzido por formigueiros de Atta sexdens rubropilosa Forel.

Os ensaios experimentais foram realizados utilizando-se oito formigueiros adultos (cinco anos) de A. sexdens rubropilosa criados no laboratório da Unidade de Estudos em Ecologia de Comunidades (UEEC) da Universidade Federal de Viçosa. As colônias foram mantidas em fotoperíodo de $12 \mathrm{~h}$ ( $12 \mathrm{~h}$ luz e $12 \mathrm{~h}$ escuro) a $26 \pm 1{ }^{\circ} \mathrm{C}$ e alimentadas com folhas frescas de Acalypha wilkesiana Müll. Arg. (Euphorbiaceae) ou Bauhinia variegata L. (Caesalpiniaceae). Cada colônia constituiu-se de um pote plástico (5 L) acoplado a um pote cerâmico $(1 \mathrm{~L})$ e a uma arena plástica de forrageamento $\left(500 \mathrm{~cm}^{2}\right)$ por meio de mangueiras de silicone $(\varnothing=2,5 \mathrm{~cm})$. Um recipiente de $100 \mathrm{~mL}$ foi 
colocado no centro da arena de forrageamento com algumas formigas mortas da colônia, condicionando as operárias a depositarem todo o lixo produzido neste recipiente. Os primeiros 60 dias serviram para o condicionamento das colônias à dieta de folhas e para que todo o lixo produzido fosse oriundo de um mesmo material vegetal. Após o período de condicionamento, foram feitas coletas diárias do material vegetal e do respectivo lixo produzido pelos formigueiros por um período de 15 dias, e essas amostras foram submetidas aos ensaios de determinação dos macronutrientes. As amostras de folha e de lixo foram secas em estufa a $70^{\circ} \mathrm{C}$, com as amostras de lixo sendo ainda peneiradas $(\varnothing=2 \mathrm{~mm})$, para remoção de formigas mortas. Ambas as amostras foram moídas em moinho para material vegetal e, a seguir, procedeu-se à digestão ácida das mesmas, para quantificar os elementos $\mathrm{N}$, $\mathrm{P}, \mathrm{K}, \mathrm{Ca}, \mathrm{Mg}$ e $\mathrm{S}$. Na determinação do N, efetuou-se a digestão sulfúrica das amostras e aplicou-se o método de Kjeldahl descrito por Malavolta et al. (1997). Quanto aos demais elementos, procedeu-se à digestão nitroperclórica. A análise do teor de $\mathrm{P}$ foi feita por espectroscopia de absorção molecular com base no método de Watanabe \& Olsen (1965). O K foi quantificado por fotometria de chama (Malavolta et al., 1997). Os teores de Ca e Mg foram determinados por meio de espectrometria de emissão óptica com plasma acoplado por indução (Silva, 1999). O teor de $\mathrm{S}$ foi obtido turbidimetricamente, seguindo-se o procedimento descrito por Alvarez et al. (2001). As diferenças nas concentrações dos macronutrientes nos diferentes tratamentos (lixo ou folhas) foram analisadas por meio de ANOVA e comparadas pelo teste $\mathrm{T}$.

As concentrações de nutrientes no lixo foram significativamente maiores do que nas folhas, independentemente do substrato (Quadro 1). As concentrações dos elementos $\mathrm{P}, \mathrm{Ca}, \mathrm{Mg}$ e $\mathrm{S}$ nas folhas de $A$. wilkesiana foram sistematicamente maiores do que as de $B$. variegata. Entretanto, as concentrações de nutrientes no lixo dos formigueiros foram muito semelhantes (Quadro 1). Assim, houve aumento dos teores de nutrientes nos lixos das colônias. Em outras palavras, os sauveiros estudados não só conservaram os valores originais nas folhas consumidas, como também adicionaram mais nutrientes ao lixo. Esse fato demonstra a importância de colônias de $A$. sexdens rubropilosa na síntese e reciclagem biológica de nutrientes. Corroborando esses dados, diversos estudos reportam que solos de sauveiros apresentam maior fertilidade quando comparados a solos adjacentes sem a influência das colônias (Farji-Brener \& Illes, 2000; Moutinho et al., 2003; Sousa-Souto et al., no prelo). Esses estudos têm demonstrado concentrações de macronutrientes 1,3 a 80 vezes maiores nos sauveiros do que em solos sem a influência das colônias e indicam que a redistribuição de materiais pelas formigas e seus efeitos nas propriedades dos solos podem desenvolver regiões de maior fertilidade em diversos ecossistemas neotropicais. Por exemplo, dados obtidos dos teores de macronutrientes de amostras de Latossolo Vermelho-Amarelo distrófico (LVAd), da região da Zona da Mata mineira, indicam variação dos teores de $\mathrm{P}$ total (P total) de 0,3 a 0,6 $\mathrm{g} \mathrm{kg}^{-1}$ (Nunes et al., 2001). Ao comparar esses valores com os encontrados no lixo dos formigueiros, pode-se observar que o valor de $\mathrm{P}$ total no lixo apresenta concentrações cerca de 10 vezes maiores, sugerindo uma importância no ciclo orgânico de P nos solos. Essa comparação entre lixo e solo é plausível, uma vez que, em condições de campo, todo o lixo produzido por espécies de saúvas no Brasil é totalmente incorporado ao solo do formigueiro (Coutinho, 1984).

Uma vez que o lixo produzido pelas saúvas apresenta alta razão lignina/celulose (Guerra et al., 2005), é possível que os nutrientes no lixo não sofram pronta mineralização, pois substratos com altos teores de lignina e celulose apresentam difícil decomposição (Norby, 1997). Assim, é possível que os nutrientes do lixo passem a envolver um ciclo mais lento de biodisponibilização de nutrientes, em condições não

Quadro 1. Teores de macronutrientes em folhas de Acalypha wilkesiana Müll. Arg. (Euphorbiaceae) e Bauhinia variegata L. (Caesalpiniaceae) e no lixo produzido por Atta sexdens rubropilosa Forel (Hymenoptera: Formicidae), a partir desses dois substratos

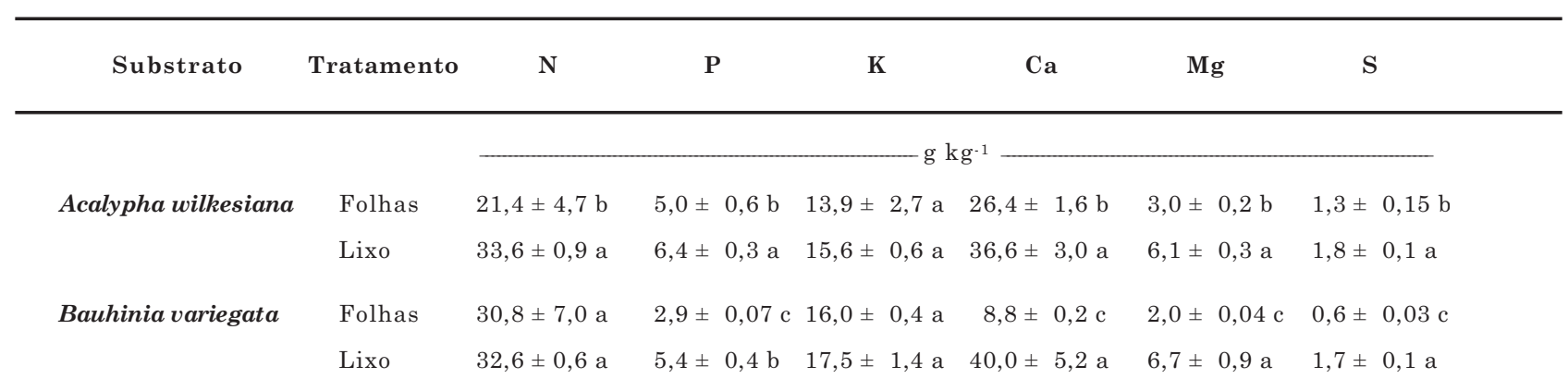

Letras diferentes na mesma coluna indicam diferença significativa $(p<0,05)$. 
favoráveis de temperatura e umidade para sua decomposição. Essa lenta biodisponibilização tornase um fator positivo, visto que permite a manutenção de estoques de nutrientes no longo prazo, garantindo suprimento mais gradual desses minerais (Novais \& Smyth, 1999). Vanegas (2005), estudando estercos in natura provenientes da criação de bovinos de leite, suínos, caprinos, ovinos, aves e coelhos de corte, caracterizou-os quimicamente por meio de ensaios de incubação. Comparando a constituição química dos resíduos orgânicos estudados por Vanegas (2005) com os teores médios encontrados em lixos de formigueiros (Quadro 1), percebem-se algumas semelhanças nos teores de alguns nutrientes. Os teores médios de $\mathrm{Mg}$ e $\mathrm{K}$ dos resíduos orgânicos (6,10 e 17,80 $\mathrm{g} \mathrm{kg}^{-1}$, respectivamente), por exemplo, foram muito próximos daqueles encontrados para os lixos dos formigueiros (6,40 $\mathrm{g} \mathrm{kg}^{-1}$ de $\mathrm{Mg}$ e 16,54 $\mathrm{g} \mathrm{kg}^{-1}$ de K). O mesmo ocorre com os outros nutrientes, como $\mathrm{N}$ e $\mathrm{P}$, cujos teores são bem próximos aos das médias dos lixos dos formigueiros. A similaridade observada entre a constituição química dos lixos e a dos resíduos orgânicos permite considerar os refugos dos formigueiros de saúvas como um potencial adubo orgânico. Considerando que a densidade de sauveiros em algumas áreas pode chegar a 50 colônias ha ${ }^{-1}$ (Araújo et al., 1997), o efeito da bioacumulação de nutrientes pode ser extremamente benéfico para o ecossistema onde tais colônias estão inseridas. Por outro lado, para que se possa estabelecer sua real contribuição na ciclagem de nutrientes em solos tropicais, são necessários estudos da degradação dos componentes do material de descarte dos sauveiros em condições ótimas. Cabe ressaltar que as concentrações de nutrientes do lixo no experimento com os formigueiros artificiais foram obtidas ao nutrir as colônias com apenas duas espécies vegetais, enquanto em condições de campo as saúvas irão cortar uma diversidade muito maior de espécies, com maior ou menor suscetibilidade a mineralizar-se e com distintas concentrações de elementos. No entanto, diante da carência de resultados analíticos nesse tipo de material, o presente trabalho torna-se um referencial importante para outros estudos complementares.

\section{CONCLUSÃO}

O lixo de colônias de $A$. sexdens rubropilosa apresenta maiores concentrações de macronutrientes quando comparadas com os teores observados nas folhas utilizadas como substrato. Esses acréscimos nas concentrações variam de 6 a $360 \%$, dependendo do substrato cortado e do nutriente analisado. Esses valores são semelhantes aos encontrados em sauveiros no campo, indicando a importância de colônias de $A$. sexdens rubropilosa na acumulação biológica de nutrientes e sua reciclagem em solos da região neotropical.

\section{LITERATURA CITADA}

ABRIL, A.B. \& BUCHER, E.H. Nutritional sources of the fungus cultured by leaf-cutting ants. Appl. Soil Ecol., 26:243-247, 2004.

ALVAREZ V., V.H.; DIAS, L.E.; RIBEIRO Jr., E.S.; SOUZA, R.B. \& FONSECA, C.A. Métodos de análises de enxofre em solos e plantas. Viçosa, MG, Universidade Federal de Viçosa 2001. 131p.

ARAÚJO, M.S.; DELLA LUCIA, T.M.C. \& MAYHÉ-NUNES, A.J. Levantamento de Attini (Hymenoptera: Formicidae) em povoamento de Eucalyptus na região de Paraopeba, Minas Gerais, Brasil. R. Bras. Zool., 14:323-328, 1997.

BUCHER, E.H.; MARCHESINI, V. \& ABRIL, A. Herbivory by leaf-cuting ants: Nutrient balance between harvested and refuse material. Biotropica, 36:327-332, 2004.

COUTINHO L.M. Aspectos ecológicos da saúva no cerrado. A saúva, as queimadas e sua possível relação na ciclagem de nutrientes minerais. Bol. Zool. USP, 8:1-9, 1984.

FARJI-BRENER, A.G. \& ILLES, A.E. Do leaf-cutting ant nests make "bottom-up" gaps in neotropical rain forests? A critical review of the evidence. Ecol. Letters, 3:219-227, 2000 .

GUERRA, M.B.B.; SOUSA-SOUTO, L.; SCHOEREDER, J.H.; SCHAEFER, C.E.G.R. \& SILVA, W.L. Produção de lixo em colônias de Atta sexdens rubropilosa (Hymenoptera: Formicidae) e sua relação com a qualidade do material vegetal cortado. In: SIMPÓSIO DE MIRMECOLOGIA, 17., Campo Grande, 2005. Anais. Campo Grande, Universidade Federal de Mato Grosso do Sul, 2005. p.419420 .

HÖLLDOBLER, B. \& WILSON, E.O. The ants. Cambridge, Harvard University Press, 1990. 732p.

MALAVOLTA, E.; VITTI, G.C. \& OLIVEIRA, S.A. Avaliação do estado nutricional de plantas - princípios e aplicações. Piracicaba, POTAFOS, 1997. 319p.

MOUTINHO, P.; NEPSTAD, D.C. \& DAVIDSON, E.A. Influence of leaf-cuting ant nests on secondary forest growth and soil properties in Amazonia. Ecology, 84:12651276, 2003.

NORBY, R. Carbon cycle - Inside the black box. Nature, 388:522-523, 1997.

NOVAIS, R.F. \& SMYTH, T.J. Fósforo em solo e planta em condições tropicais. Viçosa, MG, Universidade Federal de Viçosa, 1999. 399p.

NUNES, W.A.G.A.; KER, J.C.; SCHAEFER, C.E.G.R. \& FERNANDES-FILHO, E.I. Relação solos paisagem e gênese de alguns solos do domínio dos mares de morros, nas proximidades de Viçosa. R. Bras. Ci. Solo, 25:341-354, 2001.

SILVA, F.C. Manual de análises químicas de solos, plantas e fertilizantes. Brasília, Embrapa, 1999. 370p. 
SOUSA-SOUTO, L.; SCHOEREDER, J.H. \& SCHAEFER, C.E.G.R. Leaf-cutting ants, seasonal burning, and nutrient cycling in cerrado vegetation. Aust. Ecol. (no prelo).

VANEGAS, C.E.A. Caracterização, decomposição e biodisponibilidade de nitrogênio e fósforo de materiais orgânicos de origem animal e vegetal. Viçosa, MG, Universidade Federal de Viçosa, 2005. 143p. (Tese de Doutorado)
VERCHOT, L.V.; MOUTINHO, P.R. \& DAVIDSON, E.A. Leafcutting ant (Atta sexdens) and nutrient cycling: Deep soil inorganic nitrogen stocks, mineralization, and nitrification in Eastern Amazonia. Soil Biol. Biochem., 35:1219-1222, 2003.

WATANABE, I.S. \& OLSEN, S.R. Test of an ascorbic acid method for determining phosporus in water and $\mathrm{NaHCO}_{3}$ extracts from soils. Soil Sci. Soc. Proc., 29:677678, 1965. 